\title{
Adjoint-based sensitivity analysis for a numerical storm surge model
}

\author{
Simon C. Warder ${ }^{\mathrm{a}, *}$, Kevin J. Horsburgh ${ }^{\mathrm{b}}$, Matthew D. Piggott ${ }^{\mathrm{a}}$ \\ ${ }^{a}$ Department of Earth Science and Engineering, Imperial College London, London, SW7 2AZ, UK \\ ${ }^{b}$ National Oceanography Centre, Liverpool, L3 5DA, UK
}

\begin{abstract}
Numerical storm surge models are essential to forecasting coastal flood hazard and informing the design of coastal defences. However, such models rely on a variety of inputs, many of which will carry uncertainty, and an awareness and understanding of the sensitivity of the model outputs with respect to those uncertain inputs is necessary when interpreting model results. Here, we use an unstructured-mesh numerical coastal ocean model, Thetis, and its adjoint, to perform a sensitivity analysis for a hindcast of the $5^{\text {th }} / 6^{\text {th }}$ December 2013 North Sea surge event, with respect to the bottom friction coefficient, bathymetry and wind stress forcing. The results reveal spatial and temporal patterns of sensitivity, providing physical insight into the mechanisms of surge generation and propagation, and can also be used to estimate the uncertainty in skew surge model predictions due to uncertainty in each model input. Our results demonstrate the power of adjoint methods to gain relevant insight into a storm surge model, providing information complementary to traditional ensemble uncertainty quantification methods.

Keywords: Storm surge, Adjoint, Sensitivity analysis, Uncertainty quantification, Unstructured mesh, Finite element method
\end{abstract}

\section{Introduction}

Storm surge poses a significant hazard for coastal communities worldwide. Allowing for investment in adaptation measures (e.g. rising flood defences), global flood losses in 136 of the world's largest coastal cities have recently been estimated (Hallegatte et al., 2013) to rise from US\$6 bn per year in 2005 to US\$60-63 bn per year in 2050. Globally, the increase in extreme sea levels (Stocker et al., 2013) will result in critical flood defence thresholds being reached more frequently and therefore the risk of flooding will increase. The UK is vulnerable to storm surges, particularly along its North Sea coast; a large number of severe storms have impacted the UK in the last century (Haigh et al. 2016), with the two most severe of those events occurring in the North Sea in 1953 and 2013. The approximate economic impacts of the coastal flooding resulting from these events (using 2014 figures) were $£ 1.2$ bn and $£ 0.25$ bn respectively; the impact of the latter event was reduced through mitigation action taken after the 1953 event (Wadey et al., 2015). With

\footnotetext{
* Corresponding author

Email addresses: s.warder15@imperial.ac.uk (Simon C. Warder), kevinh@noc.ac.uk (Kevin J. Horsburgh), m.d.piggott@imperial.ac.uk (Matthew D. Piggott)
} 
continued development of the coastal zone in flood risk areas (ASC, 2014), the role of storm surge modelling remains vital.

Essential to the intelligent application of any storm surge model is an understanding of the model's sensitivity to its uncertain inputs. In a forecast scenario, the greatest model uncertainty arises from the meteorological forcing, namely the surface stress due to wind, and the atmospheric pressure gradient. For this reason, it is common to employ ensemble methods for uncertainty quantification, whereby the surge model is run multiple times, with each run using a different sample from the uncertain distribution of meteorological inputs (Flowerdew et al. 2010). While such ensemble methods provide a practical approach to uncertainty quantification within an operational forecast framework, they provide little insight into the patterns (in space and/or time) of the underlying model sensitivity, and they depend on the choice of meteorological ensemble.

An alternative approach to sensitivity analysis is provided by adjoint methods. In the context of numerical modelling, adjoint methods are used to efficiently compute gradients of model outputs with respect to model inputs, which can in principle vary in both space and time. Such methods have been used within a meteorological context since the 1980s (e.g. Hall et al. (1982)), and have a variety of applications within the field of coastal ocean modelling. Adjoint-derived sensitivities to model inputs can be used for gaining physical insight into a modelled system (e.g. Massmann (2010), Nowak (2015), Villaret et al. (2016)), or can be used within frameworks for model calibration, data assimilation and parameter estimation (e.g. Canizares et al. (1998), Chen et al. (2014), Heemink et al. (2002), Lardner et al. (1993), Li et al. (2013), Lu and Zhang (2006), Zhang et al. (2011)). Adjoint methods have also been previously applied to the analysis of storm surge model sensitivity to wind stress (Wilson et al., 2013, Warder et al., 2019).

Here, we apply a novel numerical coastal ocean model, Thetis, and its adjoint, to perform a storm surge sensitivity analysis with respect to multiple model inputs, namely the bottom friction coefficient, bathymetry and wind stress. We use the resulting sensitivities to gain physical insight into surge generation and propagation in the North Sea, and to estimate and compare the uncertainty in surge model outputs arising from each of these inputs, and at different locations in the model domain.

We first introduce the numerical model in section 2 and perform a brief model calibration in section 3 The adjoint mode of the model is described in section 4 and applied to sensitivity analysis in section 5 using the extreme December 2013 storm surge event as a case study. The results of the sensitivity analysis are discussed in section 6 and conclusions are made in section 7 .

\section{Forward numerical model}

Within this work, we model storm surges using Thetis, an unstructured-mesh finite element coastal ocean flow solver (Kärnä et al. 2018) implemented within the Firedrake finite element code generation framework (Rathgeber et al. 2016). We use Thetis in its two-dimensional configuration (Vouriot et al., 2019), which 
solves the shallow water equations (SWEs) in non-conservative form, given by

$$
\begin{aligned}
\frac{\partial \eta}{\partial t}+\nabla \cdot(H \mathbf{u}) & =0 \\
\frac{\partial \mathbf{u}}{\partial t}+\mathbf{u} \cdot \nabla \mathbf{u}+\mathbf{F}_{\mathbf{C}}+g \nabla \eta+\nabla\left(\frac{p_{a}}{\rho}\right) & =\frac{\boldsymbol{\tau}_{s}-\boldsymbol{\tau}_{b}}{\rho H}+\nabla \cdot\left(\nu_{h}\left(\nabla \mathbf{u}+\nabla \mathbf{u}^{T}\right)\right),
\end{aligned}
$$

where $\eta$ is the free surface height, $H$ is the water depth given by $H=\eta+h$ where $h$ is the bathymetry (measured positive downwards), $\mathbf{u}$ is the two-dimensional depth-averaged velocity vector, $\mathbf{F}_{\mathbf{C}}$ is the Coriolis force, $g$ is the acceleration due to gravity, $\rho$ is the water density, $\boldsymbol{\tau}_{b}$ is the bottom stress due to friction with the sea bed, and $\nu_{h}$ is the kinematic viscosity. The bathymetry $h$ is taken from the GEBCO 2014 dataset, with a minimum depth of $10 \mathrm{~m}$ applied to avoid the need for wetting and drying. Storm surge forcing is included via $p_{a}$ and $\tau_{s}$, which are the atmospheric pressure and surface stress due to wind, respectively. Within this work, we focus on the surge event of $5^{\text {th }} / 6^{\text {th }}$ December 2013; meteorological hindcast data for this event were provided by the National Oceanography Centre (personal communication 2018), and used to force the model. The bottom friction is parameterised via Manning's $n$ formulation, such that

$$
\frac{\boldsymbol{\tau}_{b}}{\rho}=\frac{g n^{2}}{H^{\frac{1}{3}}}|\mathbf{u}| \mathbf{u},
$$

where $n$ is the Manning coefficient. The surface stress due to wind is related to the wind velocity using a Charnock parameterisation, via the system of equations

$$
\begin{aligned}
\boldsymbol{\tau}_{s} & =\rho_{\text {air }}\left|\mathbf{W}_{*}\right| \mathbf{W}_{*}, \\
\mathbf{W} & =\mathbf{W}_{*} \frac{1}{\kappa} \log \frac{z}{z_{0}}, \\
z_{0} & =\frac{\alpha\left|\mathbf{W}_{*}\right|^{2}}{g}
\end{aligned}
$$

where $\rho_{\text {air }}$ is the density of air, $\mathbf{W}_{*}$ is the friction velocity, $\mathbf{W}$ the air velocity at a height $z$ above the free surface, $\kappa$ is the von Kármán constant, taken to be $0.4, z_{0}$ the surface roughness, and $\alpha$ the Charnock parameter. Tidal forcing is included by applying a Dirichlet boundary condition for the free surface height pn the open ocean boundaries, generated from eight harmonic constituents from the TPXO database Egbert and Erofeeva, 2002) (M2, S2, N2, K2, Q1, O1, P1, K1). This boundary condition is further modified by a correction calculated from the inverse barometer effect, which is applied to approximate surge generated externally to the model domain.

The governing equations $[1]$ are solved on an unstructured mesh using a $\mathrm{P}_{1}^{\mathrm{DG}}-\mathrm{P}_{1}^{\mathrm{DG}}$ finite element pair and a Crank-Nicolson timestepping scheme with a timestep of $100 \mathrm{~s}$. The mesh used within this work is shown in figure 1, and was generated using the qmesh python package (version 1.0.1) (Avdis et al., 2018), which interfaces the mesh generator Gmsh (version 2.10.1) (Geuzaine and Remacle, 2009). The mesh uses the UTM31 coordinate system, and its resolution varies from $3 \mathrm{~km}$ at the coastline to $25 \mathrm{~km}$ in the open ocean, resulting in a total of 23,120 triangular elements. Alternative meshes were tested, with results showing no strong sensitivity to mesh resolution. 


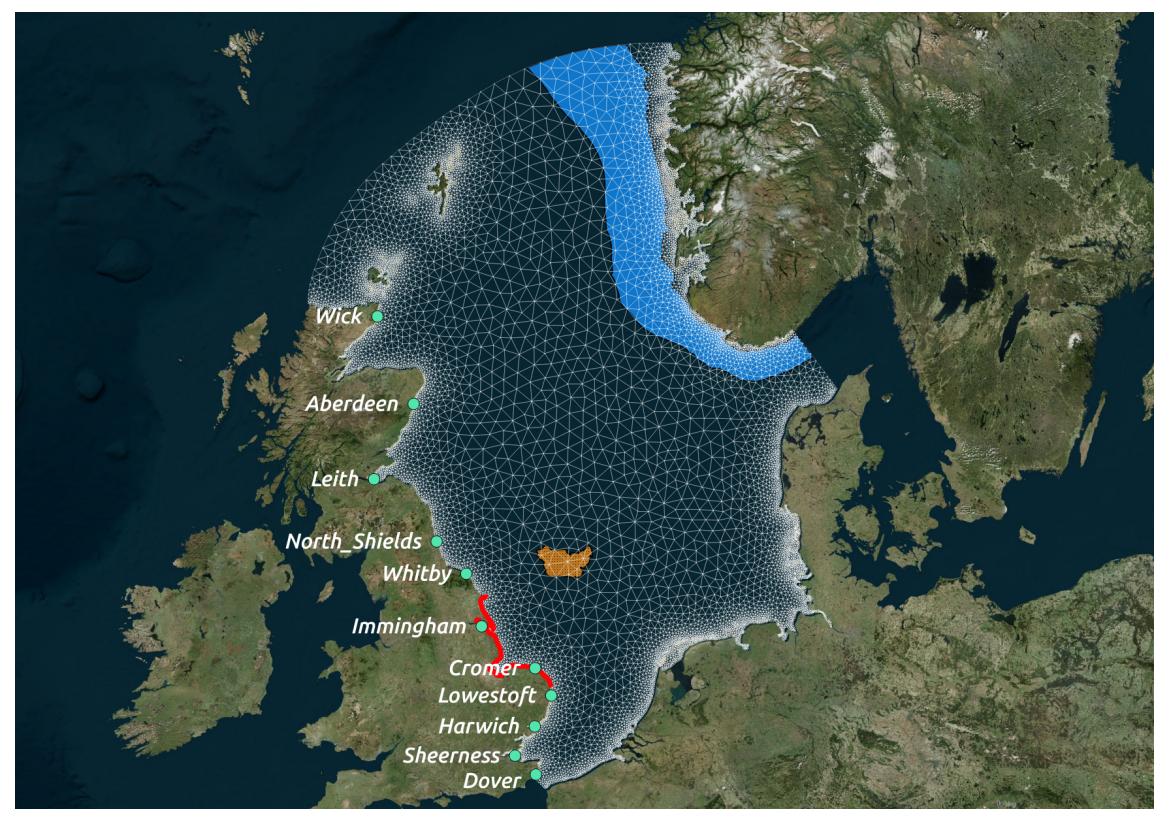

Figure 1: Mesh used for all simulations within this work, consisting of 23,120 triangular elements. Tide gauge locations shown for east coast of UK mainland. Red line indicates a section of coastline along which the average skew surge will be considered later. Two bathymetric features are highlighted for later reference: Dogger Bank (orange, extracted from $20 \mathrm{~m}$ bathymetry contour) and the Norwegian Trench (blue, extracted from 200 m bathymetry contour).

\section{Calibration}

We first calibrate the model with respect to the Manning coefficient $n$, based on a tide-only simulation. After a spin-up period of 10 days, the model is run in tide-only mode for one month, and a harmonic analysis performed at the 12 tide gauge stations within the model domain where quality controlled data is available from the British Oceanographic Data Centre (BODC), using the same eight harmonic constituents as the tidal boundary condition. The model-observation error is computed via the combined root mean squared error (RMSE) of the amplitudes of the eight harmonic constituents $C$ by

$$
\mathrm{RMSE}=\left(\frac{1}{8} \sum_{C} \frac{1}{12} \sum_{i=1}^{12}\left(A_{M, C, i}-A_{O, C, i}\right)^{2}\right)^{\frac{1}{2}},
$$

where $A_{M, C, i}$ and $A_{O, C, i}$ are the modelled and observed amplitudes of the harmonic constituent $C$ at tide gauge location $i$, respectively. The model was run as described above, for values of the Manning coefficient $n$ from $0.015 \mathrm{~s} \mathrm{~m}^{-1 / 3}$ to $0.04 \mathrm{~s} \mathrm{~m}^{-1 / 3}$ in steps of $0.0025 \mathrm{~s} \mathrm{~m}^{-1 / 3}$. The smallest value for the RMSE was 5.7 $\mathrm{cm}$, achieved with $n=0.025 \mathrm{~s} \mathrm{~m}^{-1 / 3}$. This value is used for the remainder of this paper.

In order to select an appropriate value for the Charnock parameter $\alpha$, the surge model was run for the December 2013 event using varying values of $\alpha$. For these simulations, the model is first spun up (in tide-only mode) for 10 days, prior to the wind and atmospheric pressure forcing terms being switched on approximately 10 days before the peak surge occurs. A comparison with observations for this event is made based on the modelled and observed surge residuals at the BODC tide gauge locations. As shown in figure 
2. the surge residual is defined as the difference between the total (tidally and meteorologically induced) sea surface level and the sea surface level which would be expected in the absence of surge forcing, i.e. due only to astronomical tidal forcing. For the tide gauge observations, the tidal component is computed based on a harmonic analysis of long term tide gauge data, and this harmonic part is subtracted from the surge observations to obtain the surge residual. To compute the modelled residual, the model is simply run in both full surge (tidally and meteorologically forced) and tide-only modes, and the surface elevations subtracted. For the purposes of calibrating the Charnock parameter, the model-observation error is computed by a simple root mean squared error of the residual timeseries, over a two day period capturing the peak surge, at the eight BODC tide gauges within the domain at which the surge was significant, and which recorded a sufficiently complete timeseries surface elevation record during the event. The surge model was run as described, for values of the Charnock parameter $\alpha$ from 0.01 to 0.03 , in steps of 0.002 . The smallest value for the residual RMSE was $15.9 \mathrm{~cm}$, obtained using $\alpha=0.028$, which is a value consistent with literature (Brown and Wolf, 2009).

Using these calibrated parameters, a good agreement is obtained between modelled and observed surge residuals for this event, as shown in figure 3 for the three tide gauge locations selected for the sensitivity analysis study.

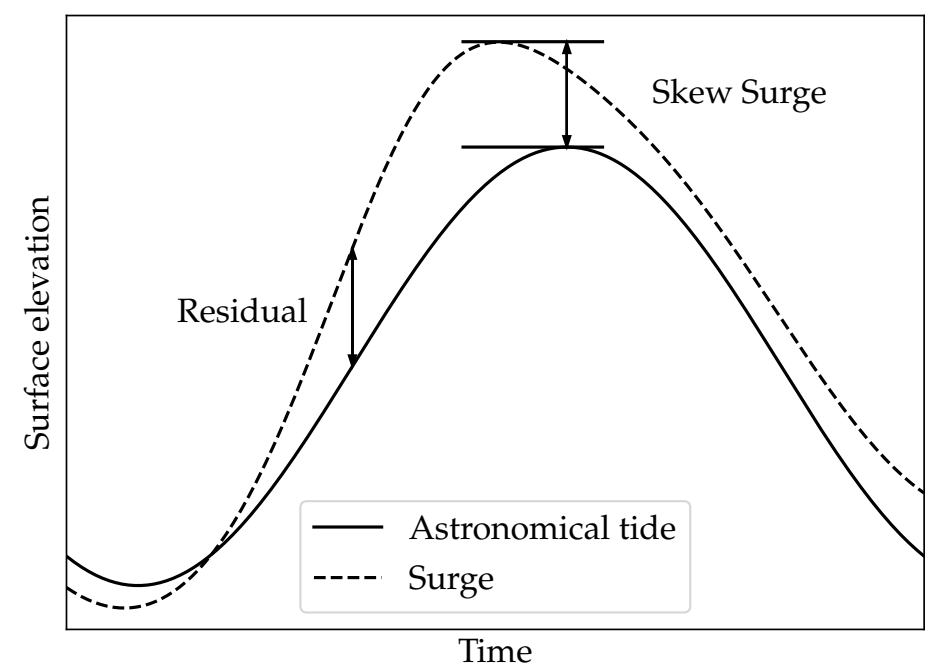

Figure 2: Schematic diagram of residual and skew surge definitions. The astronomical tide is the sea surface level which would be observed due to astronomical tidal forcing only. The surge is produced by the combination of astronomical tide with meteorological forcing.

\section{Adjoint model}

Adjoint methods can be employed when the gradient of a model output functional, $J$, with respect to a model input field $m(x, y ; t)$, is desired. In a general numerical model (which is discrete in both space 

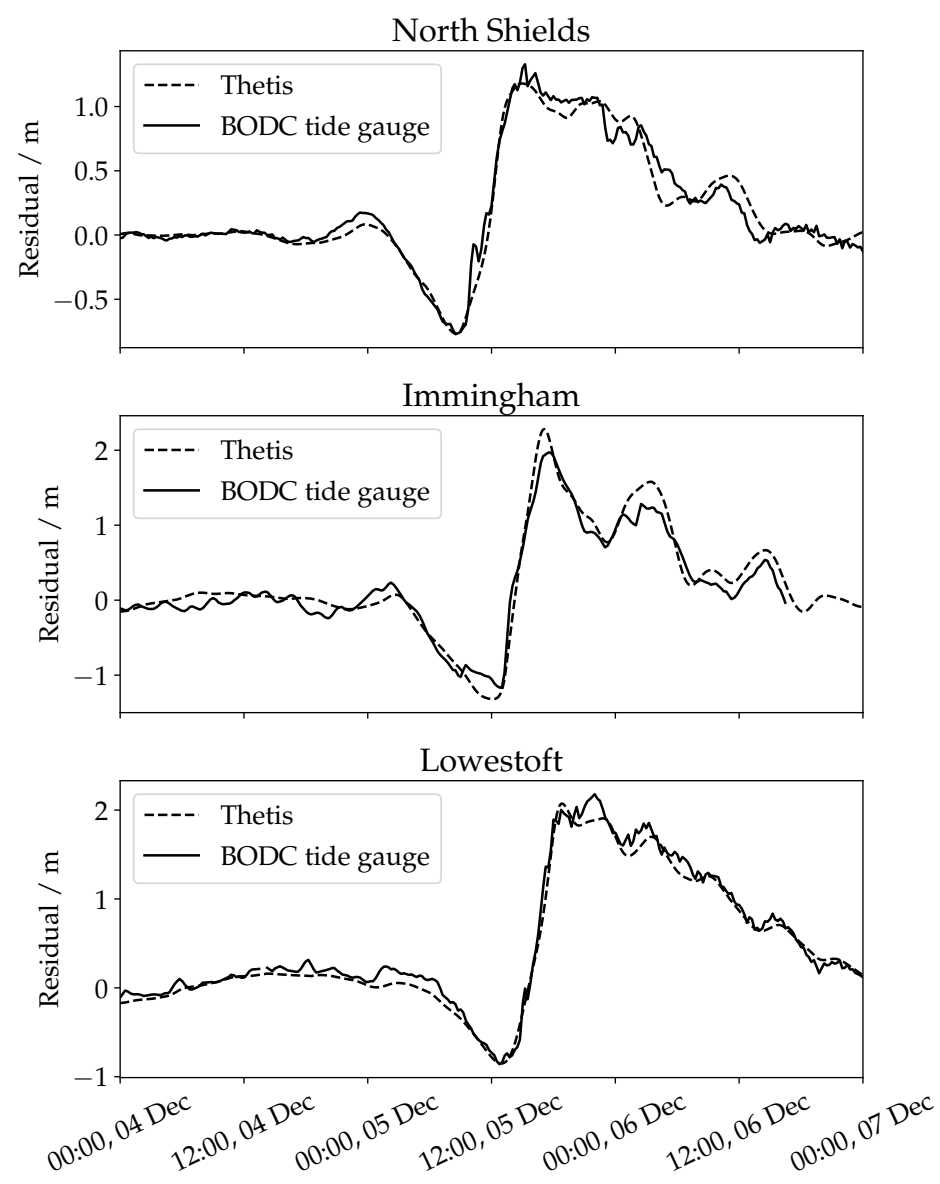

Figure 3: Comparison of modelled and observed surge residuals for the December 2013 event, at three selected tide gauge locations.

and time), these inputs are represented by a vector $\mathbf{m}$, with a sufficient number of degrees of freedom to adequately represent the full field $m(x, y ; t)$, and numerical adjoint methods can be employed to compute the gradient vector $\frac{\partial J}{\partial \mathbf{m}}$. The power of the adjoint method is that the computational cost of computing this gradient vector is approximately independent of the length of the vector, and similar to that of the forward numerical model, i.e. the approach is especially powerful when seeking the derivative of one output quantity with respect to multiple inputs. The number of degrees of freedom used to represent the model inputs can therefore be suitably large to represent a field which varies in both space and time, without impacting the computational cost. For models implemented within the Firedrake framework, the adjoint model can be generated algorithmically via the Python package pyadjoint (Mitusch et al., 2019); this package is utilised within this work to generate the Thetis adjoint model.

Storm surge model sensitivity to wind stress has been explored previously for functionals representing peak surge height at single target locations (Wilson et al., 2013, Warder et al., 2019), and the reader is referred to these works and references therein for detail on the implementation and performance of numerical adjoint 
models. Here we extend to comparisons of the sensitivity at a variety of target locations, and comparisons with other sources of uncertainty.

\section{Sensitivity to model parameters and inputs}

Taking the December 2013 surge event as a case study, we define the functional, $J$, as the skew surge at one of three single target locations (the tide gauges at North Shields, Immingham and Lowestoft), or as the mean skew surge along the section of coastline indicated in figure 1. The skew surge is defined as the maximum surge height above the astronomically (tidally) predicted high water within a tidal cycle, as shown in figure 2, and is a more relevant measure of storm surge severity than the surge residual (Williams et al. 2016). Using the adjoint model, we compute the gradient of each of these functionals with respect to three model inputs, namely bottom friction coefficient $n$, bathymetry $h$ and wind stress $\boldsymbol{\tau}_{s}$. Bottom friction coefficient and bathymetry are both scalar fields which are constant with respect to time, and the sensitivity pattern we compute with respect to these inputs is therefore only spatially varying. Wind stress is a vector field which varies in both space and time, and the sensitivity of modelled skew surges with respect to wind stress is therefore also a spatially and temporally dependent vector field.

In the cases of bottom friction coefficient and bathymetry, the skew surge sensitivity is computed in two parts, requiring one pair of forward and adjoint runs for each; the sensitivity of the peak sea surface height is computed for both the fully surge-forced and tide-only models, with the results subtracted to obtain the sensitivity of the skew surge. This is not necessary in the case of wind stress, since the tide-only peak sea surface height does not depend on the wind stress.

Model sensitivities to different inputs cannot be directly compared, since they will have incommensurable units. Instead, we estimate the uncertainty in each input, and combine this with the adjoint-derived sensitivities to estimate the contribution of each input to uncertainty in the model outputs. Since the adjoint computes the gradient $\frac{\partial J}{\partial m}(x, y ; t)$, we make an estimate of the uncertainty in the input, $\Delta m$, and perform a convolution with the sensitivity in order to estimate the uncertainty in the output $J$, given by

$$
\Delta J \approx \iiint \frac{\partial J}{\partial m}(x, y ; t) \cdot \Delta m(x, y ; t) \mathrm{d} x \mathrm{~d} y \mathrm{~d} t,
$$

which may be directly compared for different inputs $m$. Note that this is equivalent to performing a first-order Taylor expansion with respect to the input $m$.

\subsection{Sensitivity to bottom friction coefficient}

The skew surge at each target location depends on the bottom friction coefficient across the whole domain. In principle the sensitivity to bottom friction coefficient also evolves with time (i.e. the skew surge depends more strongly on the bottom friction at shorter times before the peak), but since the bottom friction coefficient is constant in time, here we remove the temporal dependence by performing the adjoint run over 
a period of approximately 10 days prior to the peak surge; longer adjoint runs were not found to affect the computed sensitivities.

The resulting fields of sensitivity to bottom friction coefficient are shown in figure 4 The greatest sensitivity magnitudes are found within relatively small regions in the vicinity of each target location. The sensitivity of the skew surge at North Shields exhibits the smallest sensitivity magnitudes, due to its position on an exposed section of coastline; the propagation of the surge as a coastally trapped wave is not strongly affected by local features, and the local value of the bottom friction coefficient therefore has only a weak effect on the skew surge at the North Shields tide gauge. The sensitivity of the skew surge at Immingham exhibits the greatest magnitudes, particularly in and around the Humber Estuary and the Wash. The dynamics of the surge propagation around this region are complex, and the waters here are particularly shallow; the $1 / H$ proportionality in the wind stress and bottom stress terms of the governing equations (1) therefore increases the model's sensitivity to bottom friction, as well as to bathymetry and wind stress, in shallow waters. This high sensitivity to the friction coefficient in the region of the Humber Estuary and the Wash is also evident for the skew surge at Lowestoft, suggesting that the interaction between the surge and this region of coastline has a lasting effect on the surge as it travels further south. Common to the sensitivity patterns for all target locations is the pattern in the far-field, i.e. in the north of the domain; this is because any effect of the bottom friction on the surge in the north of the domain is propagated with the surge as it travels south as a coastally trapped wave, and therefore has the same effect on the skew surge at all target locations.

In order to estimate the total impact of an uncertain bottom friction coefficient on model outputs via equation (5), we first estimate the uncertainty in the bottom friction coefficient ( $\Delta m$ in equation (5)). Based on typical values for the Manning coefficient (Arcement and Schneider, 1989) for the types of sediment found in the North Sea (Digimap, b), we assume an uncertainty in the Manning coefficient of $0.005 \mathrm{~s} \mathrm{~m}^{-1 / 3}$. Using equation (5) to convolve a uniform uncertainty of $0.005 \mathrm{~s} \mathrm{~m}^{-1 / 3}$ with the adjoint sensitivities shown in figure 4. we obtain an uncertainty in the skew surge of $-8.1 \mathrm{~cm}$ at North Shields, $-17.3 \mathrm{~cm}$ at Immingham and -16.1 $\mathrm{cm}$ at Lowestoft, with the minus signs indicating that increases in friction would induce reductions in skew surge due to the extraction of energy from the surge. The uncertainty in the mean skew surge along the coastline section, estimated by the same method, is $-19.9 \mathrm{~cm}$; this is of similar magnitude to the estimated uncertainties at Immingham (which is within the coastline section) and Lowestoft (just to the south of the coastline section).

\subsection{Sensitivity to bathymetry}

The sensitivities of modelled skew surges to bathymetry are shown in figure 5, where we have again performed the adjoint run over a period of approximately 10 days to remove the time dependence of the sensitivity. The observed spatial patterns share similar features to those of the sensitivity to bottom friction coefficient of figure 4. We find the greatest magnitudes of sensitivity within localised regions around each target location, and in particular we find that these localised sensitivities share similar spatial patterns with those observed for bottom friction, but with opposite signs. In the north of the domain, we again find that 

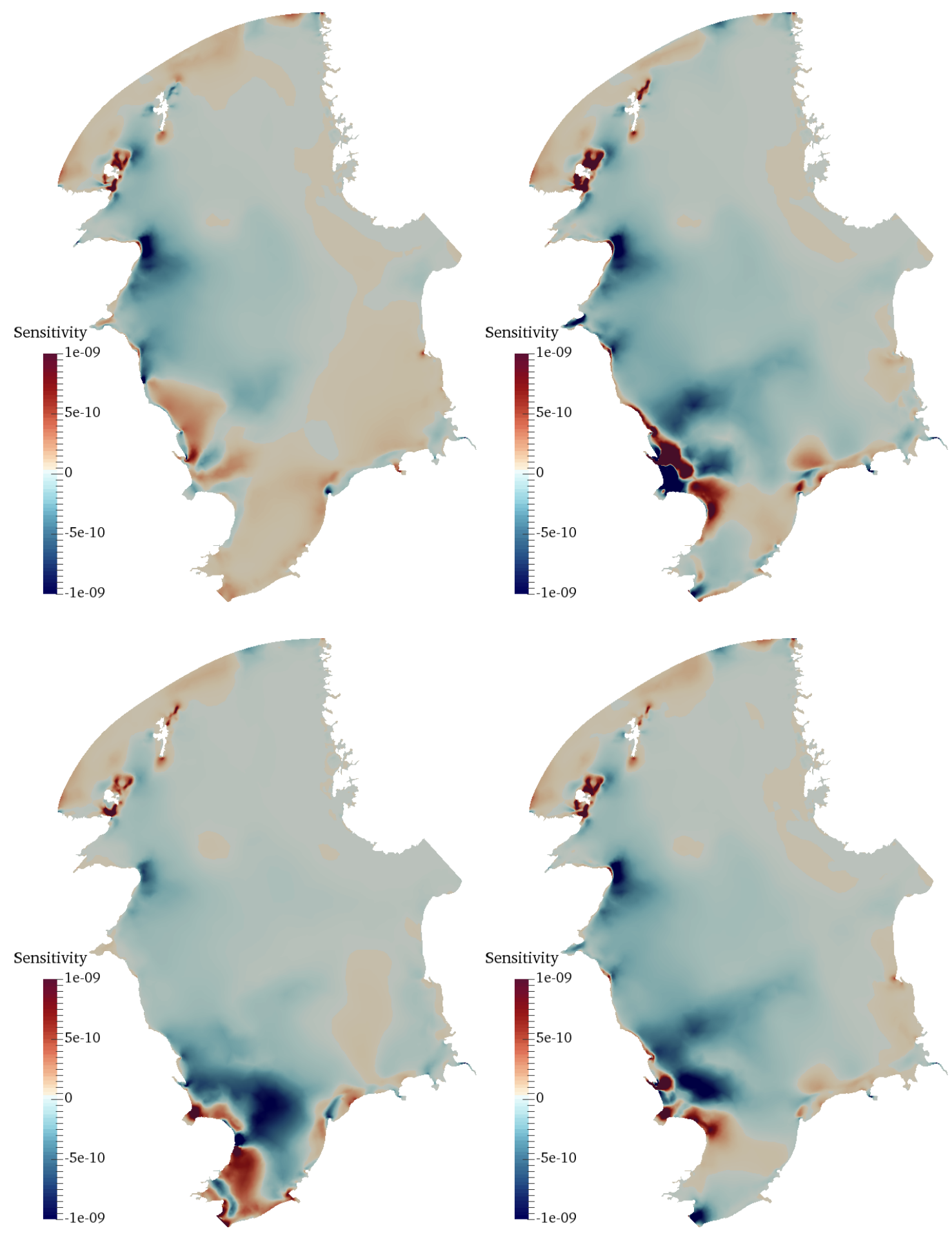

Figure 4: Sensitivity of modelled skew surges to bottom friction coefficient. Units: $\mathrm{m} \mathrm{s}^{-1} \mathrm{~m}^{1 / 3} \mathrm{~m}^{-2}$ (metres of surge, per unit Manning coefficient, per unit area). Top left: North Shields. Top right: Immingham. Bottom left: Lowestoft. Bottom right: mean along coastline section. Greatest sensitivity magnitudes are local to each location, and all locations exhibit similar patterns of sensitivity to bottom friction coefficient in the north of the domain. 
the observed patterns of bathymetry sensitivity are similar for all target locations, due to the propagation of the surge as a coastally trapped wave from north to south; any influence of the bathymetry on the surge in the north of the domain is propagated south with the surge and impacts all subsequent observation locations.

To estimate the impact of this sensitivity on model outputs, we again start by estimating the uncertainty in the bathymetry itself. For this, we compute the root mean square (RMS) difference between two bathymetric datasets. We compare the GEBCO bathymetry dataset used within the model with data from Digimap (Digimap, a), which is available at higher resolution than GEBCO, but does not cover the entire model domain. In the region of our model domain in which both GEBCO and Digimap datasets are available, the RMS difference between the two is $2.7 \mathrm{~m}$. Convolving a uniform $2.7 \mathrm{~m}$ bathymetry uncertainty with the adjoint-computed bathymetry sensitivities according to equation (5) produces skew surge uncertainties of $-2.3 \mathrm{~cm}, 6.7 \mathrm{~cm}$ and $-4.8 \mathrm{~cm}$ in the skew surges at North Shields, Immingham and Lowestoft, respectively, and $-3.7 \mathrm{~cm}$ in the mean skew surge along the coastline section. The minus signs for North Shields, Lowestoft and the coastline section indicate that an increase in bathymetry (i.e. an increase in water depth) induces a decrease in the skew surge, with the opposite being the case at Immingham.

One feature common to the bathymetry sensitivity for Immingham, Lowestoft and the coastline section is the region of positive sensitivity coinciding with Dogger Bank, to the north-east of the Humber Estuary (see figure 11. The depth of this sand bank is around $20 \mathrm{~m}$, with depths in excess of $60 \mathrm{~m}$ immediately north of the bank. The positive sign of the bathymetry sensitivity in this region indicates that an increase in bathymetry (i.e. the removal of the bank) would produce an increase in the peak residuals at Immingham, Lowestoft and the coastline section, and therefore that the bank protects the coastline to its south from the surge.

\subsection{Sensitivity to wind stress}

Wind stress and atmospheric pressure are responsible for surge generation, and the sensitivity of a surge model to these inputs therefore has the potential to provide physical insight into the surge generation mechanism. In an operational scenario, the meteorological inputs also carry high uncertainty, and understanding model sensitivity to these inputs is therefore essential to the interpretation of surge forecasts. For this surge event, we find that the contribution of atmospheric pressure to the modelled skew surge, averaged across North Shields, Immingham and Lowestoft, is less than $10 \%$ of the total, with wind stress (and some small interaction between the two) accounting for the remainder. We therefore neglect the sensitivity to atmospheric pressure, and focus instead on sensitivity to wind stress.

Since the wind stress varies in space and time, so too do the sensitivities of modelled skew surges with respect to the wind stress. Considering a model output functional corresponding to the peak surface elevation at a single location, the region of influence of the wind stress on the value of the peak elevation will expand as lead time increases, as shown in figure 6. This is due to the fact that the propagation of perturbations caused by wind stress is limited to the shallow water wave speed. For this reason, the sensitivity to wind stress can be considered as a shallow water wave propagating backwards in time, originating at the point at 

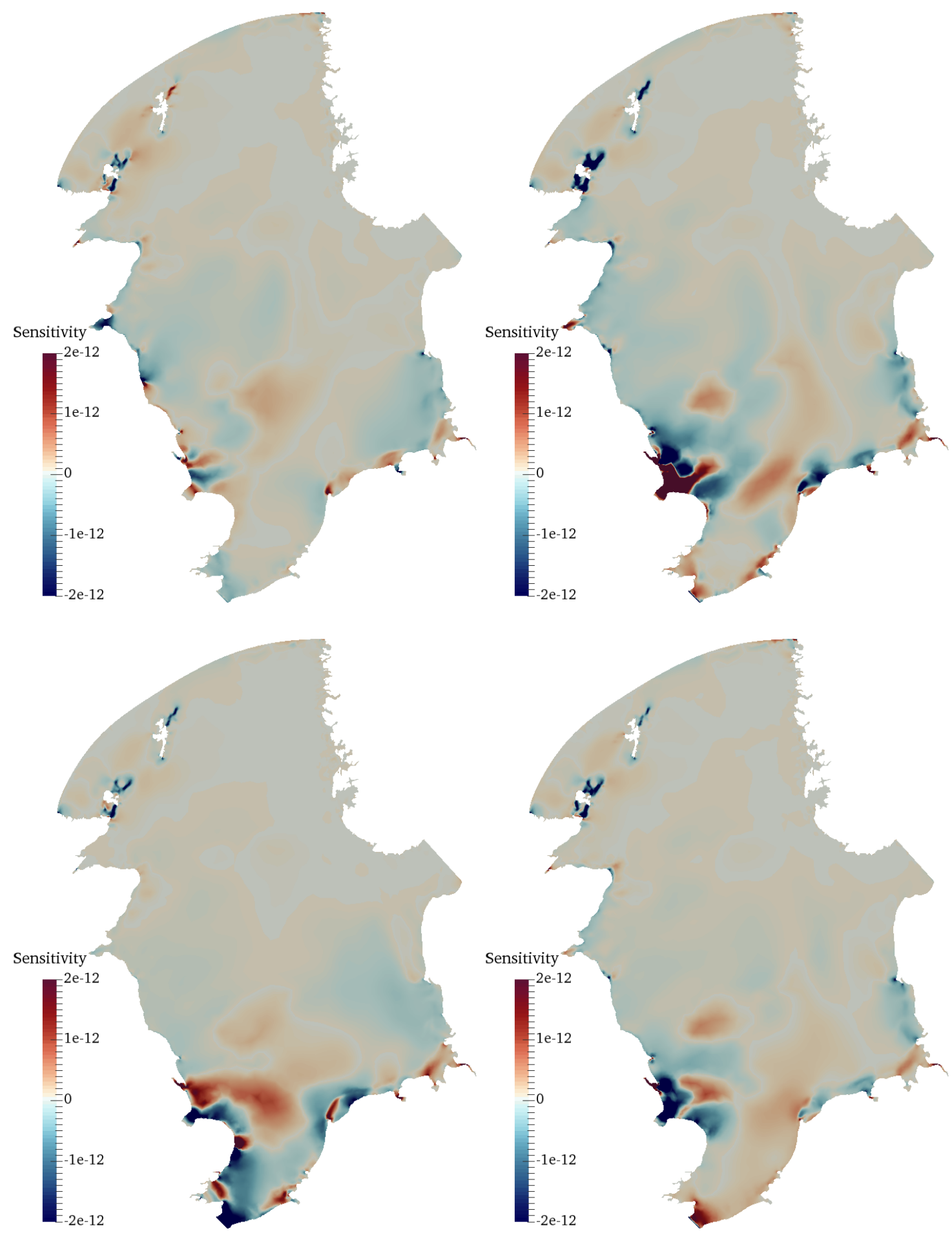

Figure 5: Sensitivity of modelled skew surges to bathymetry. Units: $\mathrm{m} \mathrm{m}^{-1} \mathrm{~m}^{-2}$ (metres of surge, per metre of bathymetry, per unit area). Top left: North Shields. Top right: Immingham. Bottom left: Lowestoft. Bottom right: mean along coastline section. The greatest magnitudes are found in the vicinity of the target locations, and the patterns in the north of the domain are similar for all plots. 

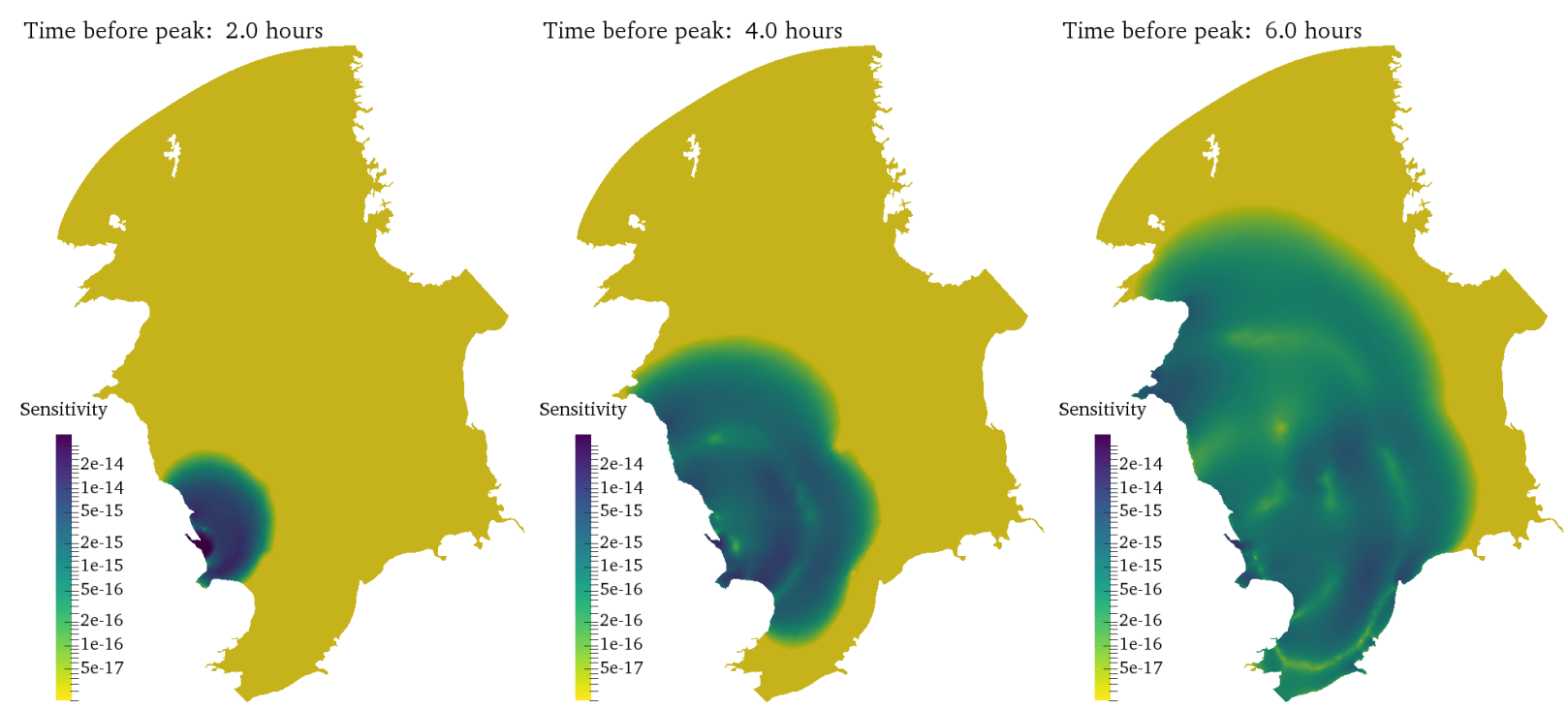

Figure 6: Snapshots of magnitude of sensitivity of skew surge at Immingham to wind stress at previous times, as labelled. Units: $\mathrm{m} \mathrm{Pa}^{-1} \mathrm{~m}^{-2} \mathrm{~s}^{-1}$ (metres of surge, per Pa wind stress, per unit area, per second). Region of influence of wind stress on skew surge increases with lead time, due to the limitation of perturbation propagation to the shallow water wave speed.

which the functional is defined. This has been explored in detail previously (Wilson et al., 2013), and can be further confirmed by an analytic approach (Warder et al. 2019).

In order to make progress comparing the wind stress sensitivities of skew surges at different locations, we can integrate the wind stress sensitivity field with respect to time to obtain an overall spatial pattern. These time-integrated sensitivities are shown in figure 7 for each target location. Similarly to the sensitivities to bottom friction coefficient and bathymetry, there are regions of high sensitivity magnitude in the vicinity of each target location, where local winds shortly before the peak surge occurs have a significant effect on the value of the peak sea surface height (and hence skew surge). All four target locations exhibit similar patterns of sensitivity to wind stress in the north of the domain, but differ more in the south, because any perturbations induced by wind stress in the north of the domain affect the coastally trapped wave which then impacts all target locations as it travels south.

The magnitudes of wind stress sensitivity are generally greater in the west of the domain. This is due to the southerly propagation of the surge along the western coastal boundary of the model domain (the east coast of the UK); winds in the east of the domain therefore have relatively little effect on the surge impacting the UK locations considered within this study. In particular, sensitivity magnitudes over the Norwegian Trench are very small. This is likely to be due to the very large depths in this region, and the $1 / H$ proportionality in the wind stress term in the governing equations (1).

Is it not possible to make a generally applicable estimate of the uncertainty associated with wind stress, since in a forecast scenario this depends strongly on the forecast lead time, and the nature of the surge event. To make a simple comparison between the overall wind stress contribution to uncertainty for each 

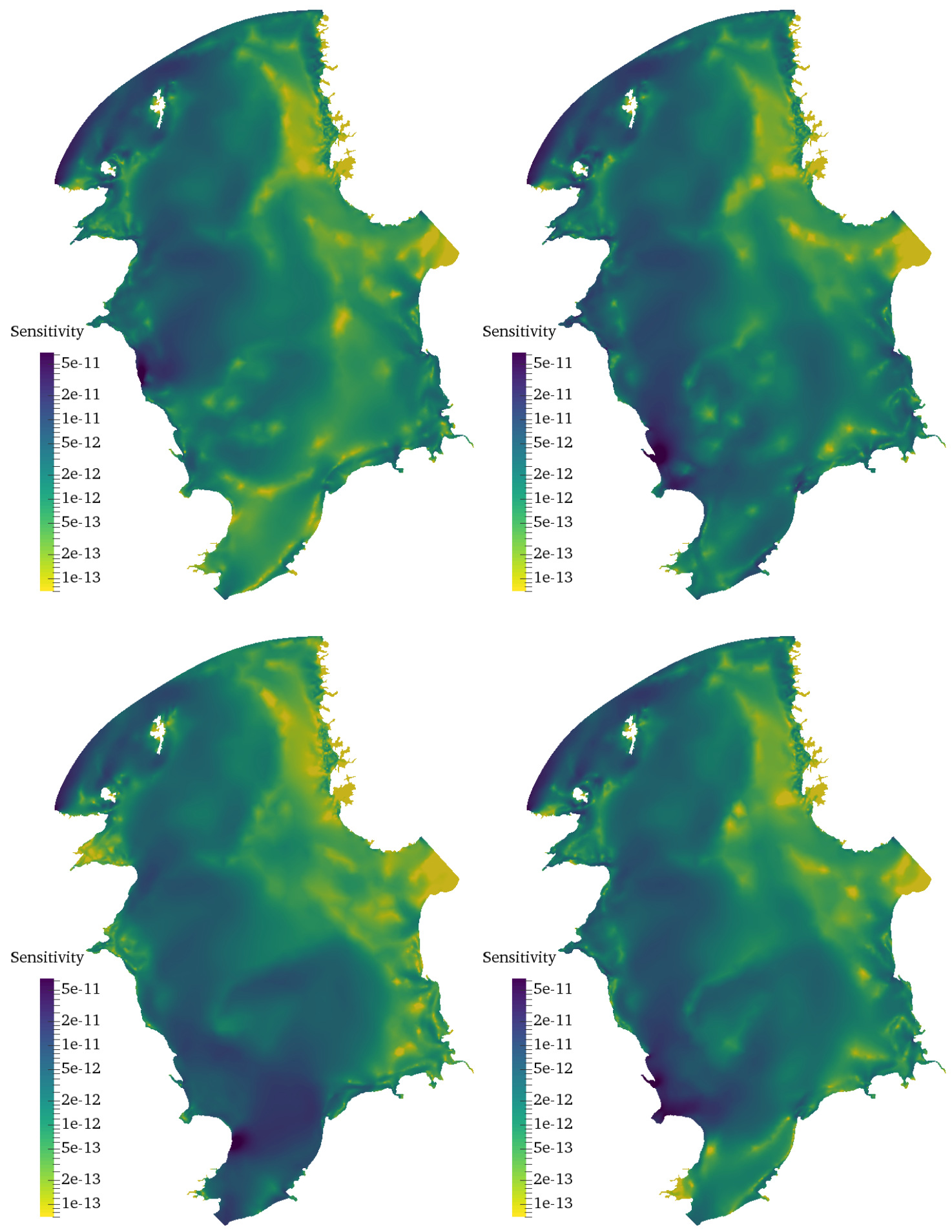

Figure 7: Magnitude of the time-integrated sensitivity of modelled skew surges to wind stress. Units: $\mathrm{m} \mathrm{Pa}^{-1} \mathrm{~m}^{-2}$ (metres of surge, per Pa wind stress, per unit area). Top left: North Shields. Top right: Immingham. Bottom left: Lowestoft. Bottom right: coastline section. Greatest magnitudes are local to each target location, and there is a similar pattern in the north of the domain for all target locations. 
target location, we therefore assume a $5 \%$ uncertainty in the wind stress magnitude, with no uncertainty in its direction. By a convolution of this uncertainty with the adjoint-derived sensitivities via equation (5), we calculate uncertainties in the skew surges of $4.3 \mathrm{~cm}$ at North Shields, $5.8 \mathrm{~cm}$ at Immingham, 7.8 $\mathrm{cm}$ at Lowestoft and $6.8 \mathrm{~cm}$ in the mean along the coastline section. This shows an increasing trend for gauges further south, due to the southward propagation of the surge and the corresponding accumulation of influence of the wind stress.

\section{Discussion}

In section 5 we used the adjoint model to explore the spatial patterns of storm surge model sensitivity to its uncertain inputs. In the cases of bottom friction coefficient and bathymetry, we have estimated uncertainties in each model input and, through convolution with the model sensitivity, estimated the resulting uncertainties in the model outputs, namely the skew surges at selected coastal target locations. In contrast to the raw sensitivities, these estimated output uncertainties can be directly compared. A summary of these estimated uncertainties is shown in table 1. We make three key observations:

(i) Estimated uncertainties due to bottom friction are of greater magnitude than those due to bathymetry. However, it should be noted that we have made very simple estimates of input uncertainties, and for a well-calibrated model these uncertainties would likely be significantly reduced. This is particularly the case for the bottom friction, and these results highlight the importance of achieving a tight constraint on bottom friction coefficient through model calibration methods.

(ii) The uncertainty contributed by the bottom friction is of smaller magnitude for the northernmost target location (North Shields) than for the locations further south, which all exhibit similar magnitudes. This pattern is explained by the accumulation of uncertainty over the propagation path of the surge along the east coast of the UK; bottom friction acts to remove energy from the surge, and this effect is therefore cumulative along the path of the surge from north to south.

(iii) In contrast, the overall contribution of uncertain bathymetry exhibits a more variable pattern across the domain, suggesting that the effect of the bathymetry on the skew surge arises through a variety of mechanisms. The similarity (with opposite signs) between the localised spatial patterns of sensitivity to bottom friction coefficient and bathymetry (figures 4 and 5 suggests that a proportion of the sensitivity to bathymetry in these regions arises from the bottom friction term of the governing equations, which is inversely proportional to the water depth. However, given the contrasting patterns of estimated uncertainty due to each input summarised in table 1, it is clear that the sensitivity to bathymetry is more complex, and must also derive significant contributions from the other terms of the governing equations (1) in which the bathymetry $h$ appears, i.e. the wind stress and surface elevation advection terms.

A direct comparison with uncertainty due to wind stress is not possible, since the uncertainty in the wind stress depends strongly on the forecast lead time. However, we know from ensemble forecasts for this 


\begin{tabular}{c|c|c|c|c} 
& North Shields & Immingham & Lowestoft & Coastline section \\
\hline Bottom friction coefficient $\left( \pm 0.005 \mathrm{~s} \mathrm{~m}^{-1 / 3}\right)$ & $\mp 8.1 \mathrm{~cm}$ & $\mp 17.3 \mathrm{~cm}$ & $\mp 16.1 \mathrm{~cm}$ & $\mp 19.9 \mathrm{~cm}$ \\
Bathymetry $( \pm 2.7 \mathrm{~m})$ & $\mp 2.3 \mathrm{~cm}$ & $\pm 6.7 \mathrm{~cm}$ & $\mp 4.8 \mathrm{~cm}$ & $\mp 3.7 \mathrm{~cm}$
\end{tabular}

Table 1: Summary of estimated skew surge uncertainties due to bottom friction coefficient and bathymetry, calculated from adjoint-derived sensitivities and estimated input uncertainties. In almost all cases, the response of the skew surge to positive perturbations in the inputs is a decrease in the skew surge, as indicated by the $\mp$ signs in the uncertainties, i.e. deeper water or increased friction results in decreased skew surges. The effect of bathymetry at Immingham is the exception, where a positive bathymetry perturbation (deeper water) results in increased skew surge.

event that uncertainty due to meteorological inputs was on the order of $1 \mathrm{~m}$ at a forecast lead time of 24 hours; this is far greater than the uncertainties due to bottom friction and bathymetry estimated here. The quantitative results of this study are therefore consistent with the perceived limitations of the operational model at the time, namely that storm surge forecast model performance is limited by the accuracy of the meteorological forecast providing the wind stress (and atmospheric pressure).

The results of an adjoint sensitivity analysis as performed within this study are highly relevant at the interface between models and observations. The spatial pattern of sensitivity to bottom friction coefficient could, for example, be used to inform the intelligent application of a spatially varying bottom friction coefficient, for the purposes of more sophisticated model calibration. For example, a choice of length scale of variation in bottom friction coefficient could be made based on the spatial variability of the model sensitivity, since variations on smaller length scales would not be constrained by observations. Similarly, the relatively localised impact of uncertain bathymetry shown here suggests that bathymetric surveys, particularly in regions prone to morphological change, could be valuable in reducing uncertainty in storm surge forecasts. Finally, the observed patterns of sensitivity to wind stress could be used to inform efforts to enhance meteorological models, by identifying regions in which uncertainty in wind stress has the greatest impact on overall surge uncertainty.

In addition to assisting in analysing surge model performance, the adjoint-based sensitivity analysis performed within this work is capable of providing physical insight into surge generation and propagation. The skew surges at Immingham, Lowestoft and the coastline section all show a positive gradient with respect to the bathymetry over Dogger Bank, to the north-east of the Humber Estuary; this is visible in figure 5 This reveals the protective effect of this bank for the south-east coast of the UK, against this storm surge event. Similarly, the sensitivity to wind stress of figure 7 shows very low sensitivity over the Norwegian Trench, due to the deep water in this region. Features such as these are simple to interpret within the physics contained in the governing equations; however, quantifying the impact of these features on the generation and propagation of the surge is non-trivial, but is achieved at relatively low computational cost by the adjoint techniques employed here.

The sensitivity analysis approach we have taken here consists of computing gradients of model outputs with respect to model inputs. This facilitates a linearisation of the model with respect to the inputs consid- 
ered, i.e. the use of a Taylor expansion as a substitute for the full forward model, via equation (5). This expansion is only valid for sufficiently small perturbations of the model inputs, but could be used, for example, to estimate an arbitrarily large ensemble of model outputs at the computational cost of only one forward and one adjoint model run (since the cost of evaluating the Taylor expansion is negligible compared to that of the full model). This is of particular interest for uncertain wind stress, where operational uncertainty quantification is typically carried out using ensemble methods. However, the viability of the adjoint-based approach as a substitute for an ensemble method is limited by two key factors. Firstly, the range of perturbations in the ensemble may exceed the linear response regime of the model, and secondly, the adjoint model must be computed separately for every model output of interest. An ensemble forecast or hazard assessment over a large spatial scale is therefore not feasible using adjoint methods alone. However, as we have shown here, an adjoint-based sensitivity analysis can provide information complementary to ensemble methods; for a given event, the adjoint in conjunction with an ensemble method could provide a more complete analysis of the potential inundation consequences for flood risk assessment purposes than ensemble methods alone.

\section{Conclusions}

In this work, we have applied adjoint methods to perform sensitivity analysis and uncertainty quantification for a storm surge model, in particular comparing the sensitivity of the modelled skew surge, at different locations across the domain, to three different model inputs, namely the bottom friction, bathymetry and wind stress. Based on the results of this work, conclusions can be drawn based on both the underlying sensitivity patterns revealed, and also the resulting estimates of model uncertainty due to each of the model inputs.

The underlying patterns of skew surge sensitivity to all model inputs considered exhibit high spatial variability, with high sensitivity magnitudes in localised regions around each target location. However, we also find that the sensitivity to model inputs in the north of the domain is similar for all target locations; i.e. perturbations in bottom friction, bathymetry or wind stress in the north of the domain have a similar impact on all target locations. This is consistent with the storm surge propagating south as a coastally trapped wave along the east coast of the UK, since any effect of the model inputs on the surge in the north of the domain will travel south with the wave and impact all locations in its path. The spatial variability of sensitivity to each input has potentially broad implications, such as the application of a spatially varying bottom friction coefficient, the commissioning of new bathymetric surveys in regions where high sensitivity aligns with high bathymetry uncertainty, or to provide feedback informing improvements of the meteorological models providing the wind stress and atmospheric pressure forcing for surge models.

Physical insight can also be gained from the patterns of surge sensitivity. For example, we see in the sensitivity to bathymetry that locations on the UK coast towards the south of the domain are protected from the surge by Dogger Bank, a large sand bank around $200 \mathrm{~km}$ off the UK coast. We also find that sensitivity to wind stress is particularly low over the Norwegian Trench, due to the very deep water. These 
are good examples of how adjoint methods can be used to gain physical insight, and form a valuable tool for analysing the impact of a storm surge event.

Using the adjoint-derived sensitivities to estimate the uncertainty in a skew surge model prediction due to typical uncertainty in each input, we find that an uncertainty of $0.005 \mathrm{~s} \mathrm{~m}^{-1 / 3}$ in the Manning coefficient produces uncertainty in the $10 \mathrm{~s}$ of $\mathrm{cm}$ in the modelled skew surge, highlighting the importance of model calibration in constraining this uncertainty. The contribution from uncertain meteorological inputs can be on the order of $1 \mathrm{~m}$ in an operational forecast scenario, far exceeding the uncertainty due to bathymetry or bottom friction, and ensemble methods remain the most practical approach to uncertainty quantification in a forecast scenario. However, we have shown here how an adjoint-based sensitivity analysis provides complementary information to an ensemble approach, providing detailed spatial and temporal information about how input uncertainty is mapped onto outputs.

\section{Acknowledgements}

This work was funded by the EPSRC Centre for Doctoral Training in Fluid Dynamics across Scales (Grant EP/L016230/1). MDP would additionally like to acknowledge EPSRC support under Grant EP/R029423/1. We thank Jane Williams of the National Oceanography Centre for her contribution of hindcast data. We also acknowledge the Research Computing Service at Imperial College London for access to computing resources. This study uses data from the National Tidal and Sea Level Facility, provided by the British Oceanographic Data Centre and funded by the Environment Agency.

\section{References}

G. J. Arcement and V. R. Schneider. Guide for Selecting Manning's Roughness Coefficients for Natural Channels and Flood Plains. Technical report, 1989.

ASC. Managing climate risks to well-being and the economy. Adaptation Sub-Committee. Committee on Climate Change. Progress report 2014., 2014.

A. Avdis, A. S. Candy, J. Hill, S. C. Kramer, and M. D. Piggott. Efficient unstructured mesh generation for marine renewable energy applications. Renewable Energy, 116:842-856, 2018. ISSN 0960-1481. doi: https:

//doi.org/10.1016/j.renene.2017.09.058. URL http://www.sciencedirect.com/science/article/pii/ S0960148117309205.

J. M. Brown and J. Wolf. Coupled wave and surge modelling for the eastern Irish Sea and implications for model wind-stress. Continental Shelf Research, 29(10):1329-1342, may 2009. ISSN 02784343. doi: 10.1016/J.CSR.2009.03.004. URL https://www.sciencedirect.com/science/article/pii/ S0278434309001010. 
R. Canizares, A. W. Heemink, and H. J. Vested. Application of advanced data assimilation methods for the initialisation of storm surge models. Journal of Hydraulic Research, 36(4):655-674, 1998. doi: 10.1080/ 00221689809498614. URL https://doi.org/10.1080/00221689809498614.

H. Chen, A. Cao, J. Zhang, C. Miao, and X. Lv. Estimation of spatially varying open boundary conditions for a numerical internal tidal model with adjoint method. Mathematics and Computers in Simulation, 97:14-38, mar 2014. ISSN 0378-4754. doi: 10.1016/J.MATCOM.2013.08.005. URL https://www.sciencedirect.com/science/article/pii/S0378475413002000.

Digimap. Marine Themes Digital Elevation Model 6 Arc Second [ASC geospatial data], Scale 1:250000, Tiles: 2062000000, 2060010060, 2060010040, 2060010020, 2060000000, 2058010080, 2058010060, 2058010040, 2058010020, 2058000000, 2056010080, 2056010060, 2056010040, 2056010020, 2056000020, 2056000000, 2054010080, 2054010060, 2054010040, 2054010020, 2054000020,2054000000, 2052010080, 2052010060, 2052010040, 2052010020, 2052000020, 2052000000, 2050010080, 2050010060, 2050010040, 2050010020, 2050000020, 2050000000, 2048010080, 2048010060, 2048010040, Updated: 25 October 2013, OceanWise, Using: EDINA Marine Digimap Service, https://digimap.edina.ac.uk, Downloaded: 2019-10-25 $12: 26: 52.575$, a.

Digimap. DiGSBS250K [SHAPE geospatial data], Scale 1:250000, Tiles: GB, Updated: 6 September 2011, BGS, Using: EDINA Geology Digimap Service, https://digimap.edina.ac.uk, Downloaded: 2019-12-05 $15: 24: 22.171, \mathrm{~b}$.

G. D. Egbert and S. Y. Erofeeva. Efficient inverse modeling of barotropic ocean tides. Journal of Atmospheric and Oceanic Technology, 19(2):183-204, 2002. ISSN 07390572. doi: 10.1175/1520-0426(2002)019<0183: EIMOBO $\rangle 2.0 . \mathrm{CO} ; 2$.

J. Flowerdew, K. Horsburgh, C. Wilson, and K. Mylne. Development and evaluation of an ensemble forecasting system for coastal storm surges. Quarterly Journal of the Royal Meteorological Society, 136:1444-1456, 2010. ISSN 00359009. doi: 10.1002/qj.648.

C. Geuzaine and J. F. Remacle. Gmsh: A 3-D finite element mesh generator with built-in pre- and postprocessing facilities. International Journal for Numerical Methods in Engineering, 79(11):1309-1331, 2009. ISSN 00295981. doi: 10.1002/nme.2579.

I. D. Haigh, M. P. Wadey, T. Wahl, O. Ozsoy, R. J. Nicholls, J. M. Brown, K. Horsburgh, and B. Gouldby. Spatial and temporal analysis of extreme sea level and storm surge events around the coastline of the UK. Scientific Data, 3:1-14, 2016. doi: doi:10.1038/sdata.2016.107.

M. C. G. Hall, D. G. Cacuci, and M. E. Schlesinger. Sensitivity Analysis of a Radiative-Convective Model by the Adjoint Method, 1982. ISSN 0022-4928. 
S. Hallegatte, C. Green, R. J. Nicholls, and J. Corfee-Morlot. Future flood losses in major coastal cities. Nature Climate Change, 3(9):802-806, 2013. ISSN 1758678X. doi: 10.1038/nclimate1979.

A. Heemink, E. Mouthaan, M. Roest, E. Vollebregt, K. Robaczewska, and M. Verlaan. Inverse 3D shallow water flow modelling of the continental shelf. Continental Shelf Research, 22(3):465-484, feb 2002. ISSN 02784343. doi: 10.1016/S0278-4343(01)00071-1. URL https://www.sciencedirect.com/science/article/ pii/S0278434301000711.

T. Kärnä, S. C. Kramer, L. Mitchell, D. A. Ham, M. D. Piggott, and A. M. Baptista. Thetis coastal ocean model: Discontinuous Galerkin discretization for the three-dimensional hydrostatic equations. Geoscientific Model Development, 11(11):4359-4382, 2018. ISSN 19919603. doi: 10.5194/gmd-11-4359-2018.

R. W. Lardner, A. H. Al-Rabeh, and N. Gunay. Optimal Estimation of Parameters for a Two-Dimensional Hydrodynamical Model of the Arabian Gulf. Journal of Geophysical Research, 98(C10):18229-18242, 1993.

Y. Li, S. Peng, J. Yan, and L. Xie. On improving storm surge forecasting using an adjoint optimal technique. Ocean Modelling, 72:185-197, dec 2013. ISSN 1463-5003. doi: 10.1016/J.OCEMOD.2013.08.009. URL https://www.sciencedirect.com/science/article/pii/S1463500313001625.

X. Lu and J. Zhang. Numerical study on spatially varying bottom friction coefficient of a 2D tidal model with adjoint method. Continental Shelf Research, 26(16):1905-1923, oct 2006. ISSN 0278-4343. doi: 10.1016/J. CSR.2006.06.007. URL https://www.sciencedirect.com/science/article/pii/S027843430600210X

S. Massmann. Sensitivities of an adjoint, unstructured mesh, tidal model on the European Continental Shelf. Ocean Dynamics, 60(6):1463-1477, dec 2010. ISSN 16167341. doi: 10.1007/s10236-010-0347-6. URL https://doi.org/10.1007/s10236-010-0347-6

S. K. Mitusch, S. W. Funke, and J. S. Dokken. dolfin-adjoint 2018.1: automated adjoints for FEniCS and Firedrake. Journal of Open Source Software, 4(38), 2019.

W. Nowak. Using algorithmic differentiation for uncertainty analysis. In 22nd Telemac 8 Mascaret User Club, pages 52-57, 2015. doi: 10.5281/zenodo.165522.

F. Rathgeber, D. A. Ham, L. Mitchell, M. Lange, F. Luporini, A. T. McRae, G. T. Bercea, G. R. Markall, and P. H. Kelly. Firedrake: Automating the finite element method by composing abstractions. ACM Transactions on Mathematical Software, 43(3), 2016. ISSN 15577295. doi: 10.1145/2998441.

T. Stocker, D. Qin, G.-K. Plattner, M. Tignor, S. Allen, J. Boschung, A. Nauels, Y. Xia, V. Bex, and P. M. (eds.). IPCC, 2013: Climate Change 2013: The Physical Science Basis. Contribution of Working Group I to the Fifth Assessment Report of the Intergovernmental Panel on Climate Change. Cambridge University Press, Cambridge, United Kingdom and New York, NY, USA, 2013. 
C. Villaret, R. Kopmann, D. Wyncoll, J. Riehme, U. Merkel, and U. Naumann. First-order uncertainty analysis using Algorithmic Differentiation of morphodynamic models. Computers 83 Geosciences, 90:144-151, may 2016. ISSN 0098-3004. doi: 10.1016/J.CAGEO.2015.10.012. URL https://www.sciencedirect. com/science/article/pii/S0098300415300777.

C. V. Vouriot, A. Angeloudis, S. C. Kramer, and M. D. Piggott. Fate of large-scale vortices in idealized tidal lagoons. Environmental Fluid Mechanics, 19(2):329-348, apr 2019. ISSN 15731510. doi: 10.1007/ s10652-018-9626-4. URL https://doi .org/10.1007/s10652-018-9626-4.

M. P. Wadey, I. D. Haigh, R. J. Nicholls, J. M. Brown, K. Horsburgh, B. Carroll, S. L. Gallop, T. Mason, and E. Bradshaw. A comparison of the 31 January1 February 1953 and 56 December 2013 coastal flood events around the UK. Frontiers in Marine Science, 2:84, 2015. ISSN 2296-7745. doi: 10.3389/fmars.2015.00084. URL https://www.frontiersin.org/article/10.3389/fmars .2015.00084.

S. C. Warder, K. J. Horsburgh, and M. D. Piggott. Understanding the contribution of uncertain wind stress to storm surge predictions. In 4th IMA International Conference on Flood Risk, Swansea, 2019.

J. Williams, K. J. Horsburgh, J. A. Williams, and R. N. Proctor. Tide and skew surge independence: New insights for flood risk. Geophysical Research Letters, 43(12):6410-6417, 2016. ISSN 19448007. doi: 10.1002/2016GL069522.

C. Wilson, K. J. Horsburgh, J. Williams, J. Flowerdew, and L. Zanna. Tide-surge adjoint modeling: A new technique to understand forecast uncertainty. Journal of Geophysical Research: Oceans, 118(10): 5092-5108, 2013. ISSN 21699291. doi: 10.1002/jgrc.20364.

J. Zhang, X. Lu, P. Wang, and Y. P. Wang. Study on linear and nonlinear bottom friction parameterizations for regional tidal models using data assimilation. Continental Shelf Research, 31(6):555-573, apr 2011. ISSN 0278-4343. doi: 10.1016/J.CSR.2010.12.011. URL https://www.sciencedirect.com/science/ article/pii/S0278434310003857. 